The Geography of Ethnoracial Birth Weight Inequalities in the United States

Kiara Wyndham Douds ${ }^{\mathrm{a}}$ and Ethan J. Raker ${ }^{\mathrm{b}}$

Forthcoming at SSM-Population Health

${ }^{\text {aNew }}$ York University

295 Lafayette Street, 4th Floor

New York, NY, 10012

Kiara.douds@nyu.edu

bUniversity of British Columbia

6303 NW Marine Drive

Vancouver, BC

Canada V6T 1Z1

ethan.raker@ubc.ca

* Corresponding Author: Kiara Wyndham Douds, kiara.douds @nyu.edu, 1-832-724-3380. For their support and helpful comments, we are indebted to Jason Beckfield, Sarah K. Cowan, and Mary Waters. We also benefited from audiences at Harvard Center for Population and Development Studies and the 2019 meeting of the Population Association of America. 


\title{
The Geography of Ethnoracial Low Birth Weight Inequalities in the United States
}

August 23, 2021

\begin{abstract}
In this article, we describe, decompose, and examine correlates of the geography of ethnoracial inequalities in low birth weight (LBW) in the United States. Drawing on the population of singleton births to U.S.-born White, Black, Latinx, and Native American parents in the first decade of the twenty-first century ( $\mathrm{N}=28.2$ million births), we calculate county-level LBW rates and rate ratios. Results demonstrate a stark racial hierarchy in which Black infants experience the most significant disadvantage, but we also document substantial local-level variation organized in what we call a regionalized patchwork of inequality, with high-disparity counties bordering low-disparity counties coupled with regional clustering. Examining the component parts of local disparities - the LBW rates for Whites and groups of color - we find strong evidence that spatial variation in ethnoracial LBW inequalities is driven by greater variation in infants of color's health across counties than by variation in Whites' health. Further, LBW rates for groups of color are only weakly to moderately correlated with Whites' LBW rates, suggesting that the same contexts can produce racially divergent health outcomes. Examining contextual factors that predict LBW disparities, we find that more segregated, socioeconomically unequal, and urban counties have larger LBW disparities. We conclude by positing an approach to health disparities that conceptualizes ethnoracial differences in health as fundamentally relational and spatial phenomena produced by systems of White advantage.
\end{abstract}

Racial and ethnic (hereafter ethnoracial) disparities in health are one manifestation of systemic inequality in the United States. Health disparities are both a consequence of ethnoracial inequality - e.g., in income, wealth, neighborhood quality, healthcare access, or experiences of racism - and a cause - e.g., poor health may limit school attendance or participation in labor markets and social life (Phelan and Link, 2015; Williams et al., 2019). Of particular concern are differences in health at the start of life, often captured by low birth weight (LBW) $(<2500 \mathrm{~g})$. LBW is an important and commonly used indicator of infant health; it predicts infant mortality, early neurodevelopmental problems, and later life 
outcomes, such as academic performance and adult health (Boardman et al., 2002; Goldenberg et al., 1996; Hummer et al., 1999; Conley and Bennett, 2001). Understanding the extent and causes of ethnoracial disparities at life's "starting gate" (Conley et al., 2003) is a critical element of research and policy agendas focused on increasing racial equity in opportunities.

Extant studies of health, including those focusing on LBW, have examined contextual factors as key causes of ethnoracial inequalities (Cagney et al., 2007; Gorman, 1999; Kane et al., 2017; Urquia et al., 2009). Theoretically, this work is motivated by an understanding that social determinants of health vary across space, as well as the fact that U.S. public health is organized through local governments (NACCHO, 2019). However, prior findings in this literature have been contradictory or have highlighted disparate factors to be important (Brazil, 2017; Ncube et al., 2016). For example, while most research concludes that neighborhood deprivation negatively impacts health (Janevic et al., 2010), some studies find null effects (Cubbin et al., 2008). These discrepancies likely have multiple causes, but a critical limitation is the fact that most contextual research focuses on singular cases - e.g., births in an individual state, county, or hospital. Given significant variation across the U.S. in factors that predict health, opposing findings may be due to differences across contexts and therefore differences in the magnitude of ethnoracial inequalities themselves.

What these inconsistencies highlight is the fact that, although our theoretical frameworks treat ethnoracial inequalities in birth outcomes as a phenomenon that is spatially determined - by factors like segregation, healthcare access, and exposure to environmental toxins - we lack a foundational and comprehensive understanding of the geography of ethnoracial birth weight inequalities across the entire U.S. While recent research has begun to examine the geography of infant mortality (Rossen et al., 2016), few studies have examined LBW among live births. That is, while we theorize that context matters, we lack knowledge of both the extent to which and how ethnoracial birth weight inequalities vary across space. This knowledge is critical both for our empirical understanding of ethnoracial inequalities as well as for our policy efforts to decrease these inequalities.

In this paper, we fill this gap by providing a comprehensive national investigation of the geography of ethnoracial LBW inequalities in the U.S.--among the first of its kind. Using the complete population of singleton birth records for the first decade of the twenty-first century (January 1, 2000 to December 31, 2009; $\mathrm{N}=28.2$ million births), we construct county-level LBW rates and disparities for four ethnoracial groups: U.S.-born Latinx, and non-Latinx White, Black, and Native American. We examine these three groups of color because of their distinct histories of racial oppression, relative population shares, and spatial histories. In addition, these three groups are analytically informative. Black-White disparities are the most-studied, and our findings can therefore be compared directly with prior work. Spatial examination of Latinx-White disparities can provide insight into the well-documented Hispanic health paradox literature (Markides and Coreil, 1986). Finally, Native American-White disparities are one of the least studied types of racial disparities, despite Native 
peoples being the original inhabitants of North America. We include Whites as the comparison group because of our conceptualization of ethnoracial inequalities as relational, which we further discuss below. Our analysis of multiple groups allows for an assessment of whether spatial variation is consistent across groups or diverges, which would suggest that different processes shape different ethnoracial inequalities in the U.S.

We examine inequalities in counties for empirical and theoretical reasons. Empirically, counties are the smallest geography at which we have national coverage of reliable natality data. Theoretically, counties are a crucial contextual level at which health is impacted because the majority of local public health departments $(70 \%)$ are organized at the county level in the U.S.. In the majority of cases, these departments are responsible for adult and child immunizations and screenings for diseases such as STIs and high blood pressure in addition to administering programs like Women, Infants, and Children (NACCHO, 2019). Further, counties are a useful geographic level to examine other factors that affect health, such as segregation, community amenities, and community features related to poverty and rurality.

Our analysis is guided by three research questions: 1 . What is the spatial extent and patterning of variation in county-level low birth weight (LBW) ethnoracial inequalities in the United States? 2. Is the spatial variation driven by county-level variation in the health of infants of color, White infants, or both? 3. What factors predict county-level LBW inequalities?

To answer the first research question, we use individual-level birth records to estimate race-specific LBW rates and rate ratios at the county-level, allowing us to quantify and visualize local disparities. To answer the second question, we analyze these LBW rates across the distribution of local LBW disparities to better understand the source of geographic variation. Then, to answer the third research question, we examine three factors commonly theorized in the literature to impact local-level ethnoracial health inequalities: segregation, socioeconomic racial inequality, and rurality. Several scholars theorize that racial residential segregation causes health inequalities due to the spatial resource hoarding it enables. Most studies - performed on small or limited geographies - have found that segregation leads to adverse birth outcomes for Black infants (Mehra et al., 2017), but some document null or mixed effects (Hearst et al., 2008). Very few studies have examined the effect of residential segregation for groups other than Black infants (Acevedo-Garcia et al., 2003). Williams and Collins argue that racial segregation is a fundamental cause of racial health inequalities (Williams and Collins, 2001). Second, following Link and Phelan's fundamental cause theory (Link and Phelan, 1995), many scholars contend that socioeconomic racial inequalities cause health inequalities. Such inequalities at a local level may cause variations in local health inequalities. Finally, we consider rurality as a potential factor in predicting spatial variation in LBW inequalities. Ethnoracial health inequalities are present in rural and urban communities, but, because rural communities differ in critical ways from urban areas that could impact health-including the built environment, economic opportunities, and healthcare infrastructure-rurality may impact ethnoracial inequalities (Burton et al., 2013; 
Albrecht et al., 1998; Baldwin et al., 2002).

Our approach makes two primary contributions to the study of ethnoracial health inequality. First, we conceptualize these inequalities as fundamentally spatial. Our approach here is guided by the insight of race and health scholars that space has always been a critical tool in the production of racial inequality in the U.S.: White colonizing settlers violently displaced and "contained" Native Americans, and, since the abolition of slavery, White oppression of Black Americans and other groups of color has occurred through an array of spatial processes, including sundown towns, de jure segregation and redlining, White flight to suburbs, urban renewal, and Jim Crow (Loewen, 2018; Massey and Denton, 1993; Rothstein, 2017). Despite this understanding, we lack systematic empirical evidence about the spatial distribution of ethnoracial inequalities in low birth weight at a local level. Reports from the National Center for Health Statistics that document rates of LBW for Black, Latinx, and White infants at the state level show considerable state-level variation in rates for the three groups (Martin et al., 2012), and recent research documents significant countylevel variation in infant mortality (Rossen et al., 2016). However, no analyses have comprehensively analyzed LBW inequalities at a level below the state, which is surprising given the many theoretical models that posit sub-state spatial features-e.g. segregation, air quality, access to healthy foods- as fundamental to health disparities. Because of the centrality of space in inequality-generating processes, we provide a foundational understanding of the extent and scope of spatial variation in these racial health inequalities at a local geography to inform policy interventions and to further build theory.

Second, we implement a relational approach to ethnoracial inequalities: ethnoracial inequalities are the result of a system of oppression that advantages White people over people of color (Bonilla-Silva, 1997; Omi and Winant, 2014). For this reason, Hicken et al. argue for a shift in research on racial inequalities in health "to a broad, systemic view that situates these inequalities within the social, economic, and political structures of societies that maintain the dominance of a single racial group" (Hicken et al., 2018: p.2). We operationalize this perspective in our analysis by analyzing local ethnoracial disparities themselves, rather than modeling average effects for each group separately. This enables critical analytical distinctions between, for instance, a low-inequality community where Whites and people of color both experience poor health outcomes and a high-inequality community where people of color disproportionately experience poor outcomes and White people disproportionately experience good outcomes. We contend that such contexts are distinct and likely require different interventions to improve health. An approach focused only on group-specific outcomes for people of color would identify these communities as the same. Relatedly, a relational perspective highlights the different historical, socioeconomic, and political experiences across groups of color and recognizes that these differences may result in distinct manifestations of racial oppression. Indeed, our analyses show distinct geographies of inequality for Black, Latinx, and Native American infants. 


\section{Data}

We use restricted-access birth certificate data for all births occurring in the United States from 2000-2009, inclusive, from the National Center for Health Statistics (NCHS). From each birth certificate, we gather five pieces of information: infant birth weight; year of birth; and the birthing parent's racial identity, nativity, and county of residence. We use the term "parent" throughout to indicate the biological parent who gives birth. Birth certificates do not ask birthing parents' gender identity, so we refrain from using gendered language in our description of the data. These data contain the full population of births in the United States with few exceptions (Brumberg et al., 2012). From January 1, 2000 to December 31, 2009, the data contain information on 39.9 million singleton births. Infant birth weight is recorded in grams, and we classify infants weighing less than $2,500 \mathrm{~g}$ as LBW.

Our analysis is limited to births to parents who are U.S.-born and who identify as non-Latinx Black, non-Latinx Native American, non-Latinx White, or Latinx. In 2003, the birth certificate form was revised and included a change that allowed for multiple racial categories to be marked by parents. However, states did not all implement the 2003 revision at the same time; by 2009, only 28 states had implemented these changes (CDC, 2009). Consequently, we exclude parents reporting multiracial identities for data consistency but acknowledge that this is an increasingly important group to study. Further, we support future research endeavors to study foreign-born groups and Asians, who are excluded from our analysis due to space constraints.

In total, approximately $4.4 \%$ of births were missing information regarding county of birth. Because our primary analysis is at the county level, we dropped these cases. Of the remaining births, $0.09 \%$ of birth certificates were missing birth weight information. A further $0.45 \%$ of birth certificates were missing birthing parent's race or nativity or were coded as multiracial. Dropping all missing cases and limiting cases to birthing parents identifying as U.S.-born Black, Latinx, Native American, or White results in a final analytic sample of $28,234,621$ singleton births.

To each county-decade observation, we link additional data from 2000 decennial census at the start of the decade. We gather several pieces of information from the Census: population counts for non-Latinx Black, non-Latinx White, non-Latinx Native American, and Latinx residents; poverty rates for each ethnoracial group; and percent rural for counties. ${ }^{1}$

\section{Measures}

We compute each ethnoracial group's rate of LBW (LBW births per 1,000 births) at the county-decade level. We also compute rate ratios (RRs) (group of color's rate of LBW divided by the White rate of LBW) at the county-decade

\footnotetext{
${ }^{1}$ Note that we combine Dade County, FL births into Miami County, FL and we use demographic data for Boulder County, $\mathrm{CO}$ for Broomfield County, $\mathrm{CO}$, which later was created primarily from Boulder.
} 
level ${ }^{2}$. To avoid unstable estimates due to small numbers of births in a county, county rates and RRs are not calculated for groups with fewer than 100 births in the county-decade, which results in various sample sizes for each countydecade RR. We use the White group as the comparison when calculating disparities because the United States is a racialized social system that advantages White individuals over those racialized as non-White in almost every arena of life, including ones that directly or indirectly affect health (Bonilla-Silva, 1997; Williams and Collins, 1995; Williams and Sternthal, 2010). Rates and rate ratios are examined to answer the first part of the first research question regarding the spatial extent of ethnoracial LBW inequalities. Rate ratios are then mapped to answer the second portion, regarding the spatial patterning of LBW inequalities. Rates and rate ratios are then presented visually - along with their ranges and variations tabularly - to answer the second research question about the source of variation for county-level variation in LBW inequalities. Finally, rate ratios are used as model outcomes, described in more detail below, to answer our third research question.

Using census tract-level data, we calculate the Isolation Index as a measure of segregation for each group of color and Whites at the county level (BlackWhite, Latinx-White, Native American-White) (Massey and Denton, 1988). The Isolation (I) index is calculated using the following equation:

$$
\mathbf{I}_{c}=\sum_{i=1}^{n}\left[\left(\frac{x_{i}}{X_{c}}\right)\right]\left[\left(\frac{x_{i}}{t_{i}}\right)\right]
$$

where $i$ indexes a census tract. Lower case letters are census tract-level measures and upper-case are for the county level. $t_{i}$ is the total population of the tract (sum of the group of color and White); $x_{i}$ and $X_{c}$ are the total populations for the group of color in the tract and county, respectively. We chose $\mathbf{I}$ over the Dissimilarity Index for theoretical and empirical reasons. Theoretically, $\mathbf{I}$ is more consistent with our focus on resource hoarding, as it is a measure of the spatial isolation a group experiences (i.e., their isolation from Whites and potentially hoarded resources) (Bell et al., 2006). I is calculated by measuring the extent to which two groups are exposed to one another or share common census tracts and ranges from 0 to 1 and can be interpreted as the probability that a randomly selected member of the group of color shares a tract with another member of that group (Massey and Denton, 1988). ${ }^{3}$ Unlike the commonly used Dissimilarity Index, the isolation index depends on relative group size. That is,

\footnotetext{
${ }^{2}$ We use rate ratios rather than rate differences for our main analyses because of their alignment with our theoretical focus on relational inequalities. However, there is debate about whether rate ratios or rate differences are preferable in certain contexts (see Kaufman (2010); Truesdale and Jencks (2016). We present rate differences in Figure 2 and we also conduct our multivariable models with crude rate differences as the outcome instead of rate ratios in Appendix Table 6. Results are substantively similar.

${ }^{3}$ In a two-group case, as we calculate here, the Isolation Index and the Exposure Index the probability that a randomly selected member of a group of color shares a tract with a White person - sum to 1. Thus, the Isolation Index and Exposure Index produce substantively similar conclusions.
} 
I takes into account whether, for instance, the group of color makes up $75 \%$ of a county vs $10 \%$ of a county, whereas the Dissimilarity Index does not. In the case of $75 \%$, it will be much harder for members of the group of color to be isolated from Whites simply by virtue of their population size, when compared to the $10 \%$ case (Massey and Denton, 1988). This feature of the Isolation Index is particularly useful when group sizes vary significantly across units, as they do here across counties in our national analysis. It is important to pick up not only spatial separation but also group size differences when examining such wide-ranging contexts. Empirically, we opt for I because preliminary analyses using both the Dissimilarity Index and $\mathbf{I}$ indicated that the Isolation Index is more strongly associated with county-level ethnoracial LBW disparities (See appendix table 4).

To measure socioeconomic ethnoracial disparities in each county, we compute poverty rate ratios (group of color's poverty rate divided by the White poverty rate). Rurality is measured by the percent of a county's population that resides outside of an urban cluster according to the census. ${ }^{4}$

For a supplementary analysis, described further below, we include in our mutlivariable models a measure of whether a county contains a Native American reservation. To create this measure, we used the U.S. Census Bureau's 2010 Census Tract to American Indian Area Relationship File, which identifies tracts that are fully or partially located on reservations. Tracts were then matched to counties using a crosswalk of 2010 Census tracts to counties obtained from the Missouri Census Data Center's 2018 Geocorr tool. Counties that contain at least one tract that contains reservation land were coded as 1 , and all other counties were coded as 0 .

\section{Multivariable Models}

We use OLS regression to estimate the relationship between three county-level spatial features and racial disparities in LBW. We use OLS because our outcome is continuous, and we cluster standard errors at the state level to account for the fact that counties within the same state are not independently and identically distributed. The outcome for each model is the difference between the log of the group of color's LBW rate and the log of the White LBW rate (equivalent to the log of the ratio). We log the ratio to account for the asymmetric nature of ratios (e.g., a one-unit change does not mean the same thing at all parts of the distribution). For each disparity, we estimate two equations, one across-state and one within-state. For each LBW disparity in county-state $c s$, we estimate the follow across-state equation:

$$
\ln \left(\frac{L B W_{m}}{L B W_{w}}\right)_{c s}=\mathbf{I}_{c s}+\ln \left(\frac{\text { Poverty }_{m}}{\text { Poverty }}\right)_{c s}+\text { Rurality }_{c s}+e_{c s}
$$

\footnotetext{
${ }^{4}$ We also tested a trichotomous variable classifying counties as (1) urban, (2) suburban, and (3) rural, but results showed significant differences only between urban and rural counties. Thus, we opted for a continuous measure of rurality.
} 
where subscript $m$ indicates groups of color and $w$ indicates Whites. I is a measure of residential segregation using the Isolation Index, and Rurality is a measure of the population proportion residing outside an urban center. Poverty captures the adult poverty rate, and poverty ratio captures economic inequality. As with the LBW rate ratio, we log the poverty ratio.

$$
\ln \left(\frac{L B W_{m}}{L B W_{w}}\right)_{c s}=\mathbf{I}_{c s}+\ln \left(\frac{\text { Poverty }}{\text { Poverty }_{w}}\right)_{c s}+\text { Rurality }_{c s}+\theta_{s}+e_{c s}
$$

Model two adds a state fixed effect indicated by $\theta_{s}$ to examine relationships within states.

\section{Results}

\section{County-level Variation in Ethnoracial LBW Disparities}

We begin by examining the spatial extent of county-level variation in LBW inequalities to answer our first research question. Table 1 reports countylevel means, standard deviations, and ranges for each disparity as well as the national-level disparity and the correlation of county-level rate ratios with rate differences. Our results reveal a stark racial hierarchy in which Black infants experience the greatest degree of disadvantage relative to White infants. In the average county, Black infants are about twice as likely to be LBW than White infants with a rate ratio $(\mathrm{RR})$ of 2.08. Latinx and Native American infants face a smaller disadvantage and are on average $20 \%$ more likely to be LBW than White infants $(\mathrm{RR}=1.21, \mathrm{RR}=1.20$, respectively). Examining standard deviations, we see that, despite having a similar mean, Native AmericanWhite disparities vary $35 \%$ more more across counties $(\mathrm{SD}=.46)$ than LatinxWhite disparities $(\mathrm{SD}=.34)$. The Native American-White standard deviation is similar to the Black-White standard deviation $(\mathrm{SD}=.46)$, yet the Black-White range is much larger $(6.02-.75=5.27)$ than the Native American-White range $(3.19-.27=2.92)$, likely due to the smaller $\mathrm{N}$ for Native American-White counties. The Latinx-White range $(2.83-.25=2.58)$ is slightly larger than the Native American-White range despite the smaller standard deviation, indicating that the Latinx-White distribution is more clustered around the mean while also having more outliers. The Black-White range is roughly double the range of the other disparities, reflecting that the overall spread of Black-White disparities is much wider across counties. In addition, mean county-level disparities for all groups exceed national-level disparities, indicating an uneven distribution of infant health across space. Finally, county-level rate ratios are highly correlated with county-level rate differences (group of color rate minus White rate), ranging from 0.83 for Black-White to 0.97 for Latinx -White.

Critically, each disparity ranges from values below one (indicating the group of color has a lower LBW rate than Whites) to well above one. For instance, though on average Black infants experience significant disadvantage at the local level, there are some counties, such as Crockett County, TN (RR=.90), in 
which they have a LBW rate advantage relative to White infants. Identifying these local contexts and understanding how they differ from high-inequality communities could be useful for identifying factors that disrupt mechanisms of ethnoracial inequality.

Finally, the varying N's for each disparity illustrate the geographic clustering of groups across U.S. counties. While $41 \%$ of counties (1,307 counties) had 100 births to both White and Black parents in the 2000s, in only $14 \%$ of counties (461 counties) did 100 births occur for both Native Americans and Whites. This reflects primarily the geographic clustering of Native Americans; in only 14 counties did 100 Native American births occur but not 100 White births.

[Table 1 about here]

We next map county-level disparities to assess their geographic distribution and patterning. Figure 1 maps the three disparities across the U.S. and displays the rate ratio percentile rank for each county. Percentile ranks are determined for each disparity separately - i.e., counties in the Black-White map are colored according to each county's percentile rank among the Black-White county disparities. Bright yellow indicates counties with the smallest rate ratios, whereas dark blue indicates counties with the largest rate ratios.

All disparities exhibit what we describe as a regionalized patchwork of inequality, with high-disparity counties bordering low-disparity counties in addition to regional clustering. These patterns provide clear evidence of the impact of local contexts on ethnoracial health inequalities. We discuss each map in turn.

The top panel of Figure 1 maps county-level Black-White LBW disparities. We see both a patchwork across the United States as well as notable regional variation. Specifically, a cluster of the largest disparities are in counties in the Northeast corridor around New York City from New Jersey to Connecticut. Many counties in the Upper Midwest also have large disparities. There is large heterogeneity in the Mississippi Delta and throughout the Sun Belt, with many instances of neighboring counties experiencing drastically different disparity levels. For instance, in Windham County, CT, there is a small disparity in LBW rates between Black and White infants $(\mathrm{RR}=1.1)$; however, in neighboring Tolland County, infants born to Black parents are 2.6 times more likely than infants of White parents to be LBW. Stark patchwork patterning like this across the U.S. suggests the importance of local factors in shaping health inequalities.

Latinx-White inequality is also regionally clustered, as seen in the middle panel of 1 . Again, counties in the Northeast have consistently large disparities. There is a patchwork pattern of inequality across counties within several states, namely Texas, North Carolina, and Illinois, where even neighboring counties exhibit strikingly different disparities. Counties in Florida have relatively uniform and small LBW disparities, while counties in the Western and Southwestern region have similarly small across-county heterogeneity with disparities near the median.

Finally, the bottom panel of Figure 1 maps Native American-White disparities. There is a cluster of smaller disparity counties in the lower Plain states, namely Oklahoma, and, to a lesser extent, in Arizona. In the West Coast and 
Southwest, there are large differences across counties, and, again in the Northeast corridor, there is a cluster of larger inequalities. Notably, isolated Native American populations - indicated by counties with no or few neighboring counties meeting our population threshold for inclusion in the analysis - tend to have high disparities, whereas in areas with larger Native populations spanning multiple counties, such as in Oklahoma, disparities tend to be smaller. Three relatively isolated counties with many births to Native Americans and with large RRs are Thurston County, NE $(R R=3.01)$, Providence County, RI $(\mathrm{RR}=2.16)$, and Corson County, SD (Standing Rock) $(\mathrm{RR}=3.19)$. However, Corson County and Thurston County had very few births to Whites compared to Native Americans. For example, 544 births to Native Americans occurred in Corson County in the 2000s, and only 112 occurred to Whites. Thurston County is home to the Omaha Tribe, and Corson County contains the Standing Rock Sioux Reservation.

[Figure 1 about here]

\section{Examining the Source of Geographic Variation in Disparities}

We turn next to our second research question: Is spatial variation in LBW inequality driven by the health of infants of color, White infants, or both? The geography of LBW inequalities documented above may be due to spatial variation in Whites' LBW rates, variation in groups of colors' LBW rates, or both. We find strong evidence that spatial variation in LBW disparities are driven by greater variation in the group of color's rates across counties than by variation in Whites' rates of LBW. The panels in Figure 2 plot county-level LBW rates for Whites and each group of color. Each county's White rate and group-of-color rate are connected by a vertical line, reflecting the rate difference of that county. Counties are ordered across the $\mathrm{X}$-axis by the size of their rate ratio (group of color rate/White rate). Each of the three panels in Figure 2 shows that, across the distributions of county-level LBW inequality (X-axis) (i.e., moving from left to right, from higher to lower disparities), the LBW rate (Y-axis) of Whites varies considerably less that of the group of color. In tabular form, Table 2 shows that the standard deviations of county-level LBW rates are greater for groups of color relative to Whites' LBW rate. Specifically, compared to White infants' rates, county-level LBW rates for Black, Latinx, and Native American infants vary $87 \%, 56 \%$, and $76 \%$ more, respectively.

[Figure 2 about here]

These patterns mean that even in the same county contexts, people of color and Whites experience different health realities. Divergent health status at the county level signals that the same contextual conditions may in part influence health outcomes for Whites and groups of color differently. Of the groups of color we examine, LBW rates for Black and Latinx infants are only moderately correlated with Whites' LBW rate $(\mathrm{r}=.43$ and $\mathrm{r}=.40$, respectively), while countylevel LBW rates for Native Americans are weakly correlated with those of White infants $(\mathrm{r}=.23)$. Thus, the factors that contribute to healthy White infants do not necessarily lead to healthy Black, Latinx, and Native American infants, and 
vice versa.

[Table 2 about here]

\section{Predictors of Geographic Variation in Disparities}

Given the geography of LBW inequality, what contextual factors influence spatial variation? Figure 3 displays the estimates from adjusted regression models predicting logged county-level group of color-White LBW disparities (i.e., the difference between the log LBW rate for the group of color and the log LBW rate for Whites) with and without state fixed effects (see appendix table 3 for full results). Independent variables are measures of segregation and rurality and a logged measure of economic inequality (the difference between the logged poverty rate for the group of color and the logged poverty rate for Whites). Examining our three factors of focus together within and across states reveals that for all three forms of racial inequality, greater residential segregation and economic inequality are associated with larger disparities in LBW rates between White infants and infants of color. With state fixed effects, a 1-point increase in segregation - that is, a move from no isolation to complete isolation - is associated with an $11.7 \%$ increase in the Black-White LBW ratio. A 1-point increase in Latinx-White segregation is associated with a $21.5 \%$ increase in the Latinx-White LBW rate ratio with state FE added. The pattern for Native American-White disparities are similar to the other groups in parameter direction - and even larger in magnitude. They are less consistent than the other models in terms of significance, but this may reflect the smaller number of counties in the Native American models than the others. One important caveat is necessary to these patterns. Supplemental results using alternative measures of segregation show that isolation is more robustly predictive of LBW disparities than spatial separation (see appendix table 4). Unlike the alternative Dissimilarity Index, our preferred isolation index considers group size, suggesting that a combination of relative group size and residential proximity matter for health.

A $1 \%$ increase in county racial economic inequality is associated with a .12\% increase in the Black-White LBW rate ratio. For the Latinx-White regression, the corresponding increase is .13\%. For the Native American-White regression, the increase is even larger - .18\% - though once state fixed effects are added the parameter fails to meet traditional standards of statistical significance.

In a supplemental analyses (see Appendix table 5), we replaced our segregation measure with an indicator for whether a county contains a reservation in the Native American-White model. Results showed that, controlling for socioeconomic inequality and rurality, counties with a reservation on average have a Native American-White LBW disparity that is $10.7 \%$ lower than counties that do not contain a reservation.

We also find evidence of smaller disparities in LBW rates for all groups of color in more rural places. Supplemental analyses not shown included a control for the logged county-level population of women aged 15-44, and in these models rurality is no longer significant. We do not include the logged population measure in our main models because of collinearity with our measure of rurality. 
However, the collinearity suggests that rurality is capturing elements of both density and group size. We reflect on the meaning of these findings further in the discussion.

States explain $12.9 \%$ of the county variation in Black-White inequality, $17.8 \%$ of the variation in Latinx-White inequality, and $24.0 \%$ of the variation in county-level disparities between Native American and White infant LBW rates. In the models that add our three spatial factors, the $\mathrm{R}^{2}$ estimates increase to $19.3 \%$ for Black-White LBW inequality, $21.7 \%$ for Latinx-White LBW inequality, and $27.4 \%$ of Native American-White inequality in LBW.

[Figure 3 about here]

\section{Discussion}

An important indicator of an infant's health at birth, LBW predicts mortality in the first year of life, a child's academic performance, and adult health (Boardman et al., 2002; Conley and Bennett, 2001; Hummer et al., 1999). Disparities across ethnoracial groups in LBW indicate racial inequality from the first moments of life. Understanding the scope and causes of inequality at birth is a critical research agenda, and with this article we contribute to this effort by documenting for the first time a comprehensive portrait of local ethnoracial LBW inequalities in the United States.

Examining county-level local disparities, we document substantial geographic variation in Black-White, Latinx-White, and Native American-White inequalities. Overall, our findings reveal a stark racial hierarchy in which Black infants on average experience far greater disadvantage relative to White infants than Latinx and Native American infants. Averages mask heterogeneity, however, and all disparities we analyze range from counties in which the group of color has a LBW advantage relative to Whites to significant disadvantage. This geographic variation can be leveraged in future work to better understand the causes of ethnoracial health inequalities. For instance, in $27 \%$ of counties, Latinx infants have lower LBW rates than White infants; the patterns of the Hispanic health paradox are thus present in some contexts but not others.

We also document a common spatial arrangement of disparities for all groups that we call a regionalized patchwork of inequality. Regional clustering highlights the importance of supra-local factors in shaping racial health inequalities; neighboring counties displaying vastly different disparities highlight the role of local processes and governments. Certainly the factors that shape health occur on multiple levels; what our findings make clear is the spatial nature of ethnoracial health inequalities. The COVID-19 pandemic has underscored the importance of county-level governance in public health, as county public health departments across the nation are tasked with mounting COVID-19 response strategies and policies. Our empirical findings here suggest that, in addition to county-level processes like segregation or educational opportunities, county governments may play a critical role in birth outcomes as well.

Despite overall similarity in type of geographic patterning, the disparities 
we study differ from one another in their exact spatial layout. For instance, in Terrebonne Parish, LA, Latinx infants experience a LBW advantage relative to White infants $(R R=.70)$, Native American infants experience a small disadvantage $(R R=1.12)$, and Black infants experience a large disadvantage $(R R=1.93)$. What are the community conditions that are detrimental for the health of some groups, but not others? At the same time, every group of color experiences severe disadvantages in the Northeast. What factors worsen health outcomes for all groups of color there? Our findings provide us with the information needed to ask such questions.

When we examine the source of geographic variation in disparities, we find that the substantial variation that we document is driven more by spatial variation in groups of color's health than White infants' health. Put another way: The health of Black, Latinx, and Native American infants is more variable across space than the health of White infants. And, further, the health of White infants is only weakly to moderately correlated with infants of color's health. Communities that are healthier for White infants are not necessarily healthier for infants of color. The same contexts can produce divergent health landscapes for different ethnoracial groups. We interpret these findings as further evidence that racism is a "fundamental cause" of health (Phelan and Link, 2015). That is, because laws, institutions, social rules, and opportunities are structured in the U.S. to advantage White people over people of color, divergent health outcomes even within the same communities are expected.

In what contexts are Whites less able to hoard resources or isolate themselves from experiencing the effects of disamenities or other factors that harm health? Our multivariable analyses provide some insight into the mechanisms that may contribute to White advantage. In counties that are more racially segregated and socioeconomically unequal, ethnoracial disparities are larger. In these communities, Whites may be better able to implement spatial resource hoarding - e.g., locations of hospitals, parks, and toxic waste sites - and other types of resource hoarding - e.g., political representation or other positions of power. These findings are consistent with the relative deprivation hypothesis (Walker and Smith, 2002), whereby relational features-i.e., both spatial and socioeconomic ordering-are significantly associated with greater relative difference in infant health. In addition, our analysis indicates that disparities are greater in urban areas than rural areas. Many of our theoretical accounts of mechanisms for health disparities, such as segregation, were developed using urban samples and with urban landscapes in mind. Is it more difficult to spatially hoard resources in less dense rural areas with smaller populations? Further, our supplemental analysis revealed that counties containing reservations have lower Native American-White disparities. Our analysis cannot parse the mechanisms behind this association, but two possibilities are that greater co-ethnic support or access to healthcare through the Indian Health Service result in better outcomes for Native American infants born on or near reservations. We hope future research investigates these questions and others, including more clearly specifying relationships among related mechanisms through causal mediation analysis. 
Through this work, we advance a conceptual approach to health disparities as spatial and relational phenomena produced by systems of White advantage. We take seriously the role of space by assessing county-level disparities in LBW across the entire U.S. Determining the scope and extent of geographic heterogeneity in LBW inequalities provides clear evidence of the spatial nature of racial inequality and lends insight into the ways that spatial processes shape health inequalities. By analyzing county-level disparities between groups of color and Whites, we also approach racial inequality as a relational outcome of a system of power. Much previous work misses this by only assessing health outcomes for groups of color (Bell et al., 2006; Shaw et al., 2010). We gain the ability to analytically distinguish between local contexts that are unhealthy or healthy for all, for instance, and those that are disproportionately unhealthy for people of color. For instance, in Marion County, Florida, the LBW rate of Latinx infants (57.2) is nearly equivalent to and even slightly lower than the rate for White infants (58.3) $(\mathrm{RR}=0.98)$. In Multnomah County, Oregon, Latinx infants also have a LBW rate of 57.2; however, here they are at a stark health disadvantage relative to White infants (White rate $=42.6, \mathrm{RR}=1.43$ ). Thus, Multnomah County exhibits local ethnoracial inequality in LBW and Marion County does not. Distinguishing between these types of counties, where groups of color exhibit the same LBW rate but there are different conditions of local inequality, is vital, as different processes may underlie health outcomes in these communities. For example, while resources may be unequally distributed across groups in Multnomah County, the distribution may be more equitable in Marion County.

Our empirical contributions expand our theoretical understanding of ethnoracial infant health disparities and suggest new avenues of research. For instance, the significant geographic heterogeneity we document should be incorporated into hypotheses and theories about the origins and mechanisms of health disparities. In addition, our analytic approach advances a theoretical treatment of local ethnoracial inequality as (1) relational, i.e. a function of both the White population and populations of color and as (2) heterogeneous, i.e. different across groups due to variation in the histories and experiences of racialized groups. By documenting the degree of spatial variation in ethnoracial inequalities at the very start of life, our findings equip researchers and policymakers with tools to understand and to identify potential mechanisms to mitigate these impactful inequalities. 


\section{References}

Acevedo-Garcia, Dolores, Kimberly A. Lochner, Theresa L. Osypuk, and Sukanya V. Subramanian. 2003. "Future directions in residential segregation and health research: a multilevel approach." American journal of public health 93:215-221. Publisher: American Public Health Association.

Albrecht, Stan L., Leslie L. Clarke, and Michael K. Miller. 1998. "Community, Family, and Race/Ethnic Differences in Health Status in Rural Areas 1." Rural sociology 63:235-252. Publisher: Wiley Online Library.

Baldwin, Laura-Mae, David C. Grossman, Susan Casey, Walter Hollow, Jonathan R. Sugarman, William L. Freeman, and L. Gary Hart. 2002. "Perinatal and infant health among rural and urban American Indians/Alaska Natives." American Journal of Public Health 92:1491-1497. Publisher: American Public Health Association.

Bell, Janice F., Frederick J. Zimmerman, Gunnar R. Almgren, Jonathan D. Mayer, and Colleen E. Huebner. 2006. "Birth outcomes among urban African-American women: a multilevel analysis of the role of racial residential segregation." Social Science \& Medicine (1982) 63:3030-3045.

Boardman, Jason D., Daniel A. Powers, Yolanda C. Padilla, and Robert A. Hummer. 2002. "Low Birth Weight, Social Factors, and Developmental Outcomes among Children in the United States." Demography 39:353-368.

Bonilla-Silva, Eduardo. 1997. "Rethinking racism: Toward a structural interpretation." American Sociological Review 62:465-480.

Brazil, Noli. 2017. "Spatial Variation in the Hispanic Paradox: Mortality Rates in New and Established Hispanic US Destinations." Population, Space and Place 23:e1968.

Brumberg, Heather L., Donna Dozor, and Sergio G. Golombek. 2012. "History of the birth certificate: from inception to the future of electronic data." Journal of Perinatology 32:407.

Burton, Linda M., Daniel T. Lichter, Regina S. Baker, and John M. Eason. 2013. "Inequality, family processes, and health in the "new" rural America." American Behavioral Scientist 57:1128-1151. Publisher: Sage Publications Sage CA: Los Angeles, CA.

Cagney, Kathleen A., Christopher R. Browning, and Danielle M. Wallace. 2007. "The Latino Paradox in Neighborhood Context: The Case of Asthma and Other Respiratory Conditions." American Journal of Public Health 97:919925.

CDC. 2009. "User Guide to the 2009 Natality Public Use File." Technical report. 
Conley, Dalton and Neil G. Bennett. 2001. "Birth Weight and Income: Interactions across Generations." Journal of Health and Social Behavior 42:450465.

Conley, Dalton, Kate W. Strully, Neil G. Bennett, and Neil G. Bennett. 2003. The starting gate: Birth weight and life chances. Univ of California Press.

Cubbin, Catherine, Kristen Marchi, Michael Lin, Thomas Bell, Helen Marshall, Curt Miller, and Paula Braveman. 2008. "Is neighborhood deprivation independently associated with maternal and infant health? Evidence from Florida and Washington." Maternal and child health journal 12:61-74. Publisher: Springer.

Goldenberg, Robert L., Suzanne P. Cliver, Francis X. Mulvihill, Carol A. Hickey, Howard J. Hoffman, Lorraine V. Klerman, and Marilyn J. Johnson. 1996. "Medical, psychosocial, and behavioral risk factors do not explain the increased risk for low birth weight among black women." American Journal of Obstetrics and Gynecology 175:1317-1324.

Gorman, Bridget K. 1999. "Racial and ethnic variation in low birthweight in the United States: individual and contextual determinants." Health 83 Place 5:195-207.

Hearst, Mary O., J. Michael Oakes, and Pamela Jo Johnson. 2008. "The effect of racial residential segregation on black infant mortality." American Journal of Epidemiology 168:1247-1254.

Hicken, Margaret T., Nicole Kravitz-Wirtz, Myles Durkee, and James S. Jackson. 2018. "Racial inequalities in health: framing future research." Social science $\mathscr{E}$ medicine (1982) p. 11. Publisher: NIH Public Access.

Hummer, Robert A., Monique Biegler, Peter B. De Turk, Douglas Forbes, W. Parker Frisbie, Ying Hong, and Starling G. Pullum. 1999. "Race/Ethnicity, Nativity, and Infant Mortality in the United States." Social Forces 77:1083-1117.

Janevic, Teresa, Cheryl R. Stein, David A. Savitz, Jay S. Kaufman, Susan M. Mason, and Amy H. Herring. 2010. "Neighborhood deprivation and adverse birth outcomes among diverse ethnic groups." Annals of epidemiology 20:445-451. Publisher: Elsevier.

Kane, Jennifer B., Gandarvaka Miles, Jennifer Yourkavitch, and Katherine King. 2017. "Neighborhood context and birth outcomes: Going beyond neighborhood disadvantage, incorporating affluence." SSM - Population Health 3:699-712.

Kaufman, Jay S. 2010. "Toward a more disproportionate epidemiology." Epidemiology 21:1-2. 
Link, Bruce G. and Jo Phelan. 1995. "Social conditions as fundamental causes of disease." Journal of health and social behavior pp. 80-94. Publisher: JSTOR.

Loewen, James W. 2018. Sundown towns: A hidden dimension of American racism. The New Press.

Markides, Kyriakos S. and Jeannine Coreil. 1986. "The health of Hispanics in the southwestern United States: an epidemiologic paradox." Public Health Reports 101:253-265.

Martin, Joyce A., Brady E. Hamilton, Stephanie J. Ventura, Michelle J. K. Osterman, Elizabeth C. Wilson, and T. J. Mathews. 2012. "Births: final data for 2010." National Vital Statistics Reports: From the Centers for Disease Control and Prevention, National Center for Health Statistics, National Vital Statistics System 61:1-72.

Massey, Douglas S. and Nancy A. Denton. 1988. "The dimensions of residential segregation." Social forces 67:281-315.

Massey, Douglas S. and Nancy A. Denton. 1993. American apartheid: Segregation and the making of the underclass. Harvard University Press.

Mehra, Renee, Lisa M. Boyd, and Jeannette R. Ickovics. 2017. "Racial residential segregation and adverse birth outcomes: A systematic review and meta-analysis." Social Science 83 Medicine 191:237-250.

NACCHO. 2019. "2019 National Profile of Local Health Departments." Technical report, National Association of County and City Health Officials.

Ncube, Collette N., Daniel A. Enquobahrie, Steven M. Albert, Amy L. Herrick, and Jessica G. Burke. 2016. "Association of neighborhood context with offspring risk of preterm birth and low birthweight: A systematic review and meta-analysis of population-based studies." Social Science $\mathscr{E}$ Medicine 153:156-164.

Omi, Michael and Howard Winant. 2014. Racial formation in the United States. Routledge.

Phelan, Jo C. and Bruce G. Link. 2015. "Is racism a fundamental cause of inequalities in health?" Annual Review of Sociology 41:311-330. Publisher: Annual Reviews.

Rossen, Lauren M., Diba Khan, and Kenneth C. Schoendorf. 2016. "Mapping geographic variation in infant mortality and related Black-White disparities in the US." Epidemiology (Cambridge, Mass.) 27:690. Publisher: NIH Public Access.

Rothstein, Richard. 2017. The color of law: A forgotten history of how our government segregated America. Liveright Publishing. 
Shaw, Richard J., Kate E. Pickett, and Richard G. Wilkinson. 2010. "Ethnic density effects on birth outcomes and maternal smoking during pregnancy in the US linked birth and infant death data set." American Journal of Public Health 100:707-713. Publisher: American Public Health Association.

Truesdale, Beth C. and Christopher Jencks. 2016. "The health effects of income inequality: averages and disparities." Annual Review of Public Health $37: 413-430$.

Urquia, Marcelo L., John W. Frank, Richard H. Glazier, Rahim Moineddin, Flora I. Matheson, and Anita J. Gagnon. 2009. "Neighborhood Context and Infant Birthweight Among Recent Immigrant Mothers: A Multilevel Analysis." American Journal of Public Health 99:285-293.

Walker, Iain and Heather J. Smith. 2002. Relative deprivation: Specification, development, and integration. Cambridge University Press.

Williams, David R. and Chiquita Collins. 1995. "US Socioeconomic and Racial Differences in Health: Patterns and Explanations." Annual Review of Sociology 21:349-386.

Williams, David R. and Chiquita Collins. 2001. "Racial residential segregation: a fundamental cause of racial disparities in health." Public health reports Publisher: SAGE PublicationsSage CA: Los Angeles, CA.

Williams, David R., Jourdyn A. Lawrence, and Brigette A. Davis. 2019. "Racism and health: evidence and needed research." Annual review of public health 40:105-125. Publisher: Annual Reviews.

Williams, David R. and Michelle Sternthal. 2010. "Understanding Racial/ethnic Disparities in Health: Sociological Contributions." Journal of health and social behavior 51:S15-S27. 


\section{Tables}

Table 1: County-Level Ethnoracial Low Birth Weight Rate Ratios, 2000-2009

\begin{tabular}{lcccccc}
\hline Ethnoracial Groups & Mean & SD & Range & N & $\begin{array}{l}\text { National } \\
\text { Mean }\end{array}$ & $\begin{array}{c}\rho \text { (w/ Rate } \\
\text { Difference) }\end{array}$ \\
\hline Black-White & 2.08 & 0.46 & $0.75-6.02$ & 1307 & 1.98 & 0.83 \\
Latinx-White & 1.21 & 0.34 & $0.25-2.83$ & 1062 & 1.10 & 0.97 \\
Nat. Amer.-White & 1.20 & 0.46 & $0.27-3.19$ & 461 & 1.03 & 0.95 \\
\hline
\end{tabular}

Table 2: County-Level Low Birth Weight Rates (per 1,000) by Ethnoracial Group and Disparity, 2000-2009

\begin{tabular}{lccccc}
\hline Ethnoracial Groups & White Mean & White SD & GOC Mean & GOC SD & N \\
\hline Black-White & 58.47 & 11.98 & 118.78 & 22.44 & 1307 \\
Latinx-White & 54.20 & 11.66 & 64.47 & 18.15 & 1062 \\
Nat. Amer.-White & 51.78 & 11.67 & 59.75 & 20.56 & 461 \\
\hline
\end{tabular}

Note: GOC refers to "group of color." 
Figures 
Black-White Low Birth Weight Rate Ratio $(\mathrm{N}=1,307)$

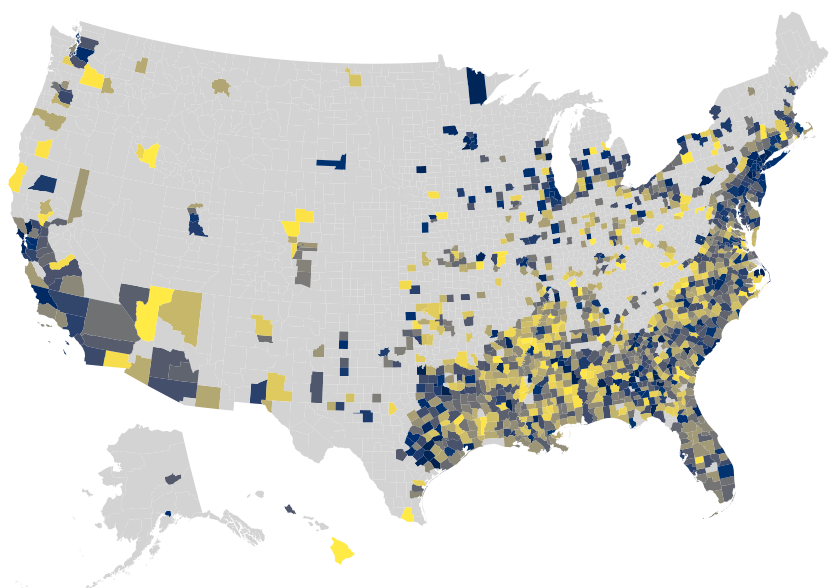

Latinx-White Low Birth Weight Rate Ratio (N=1,062)

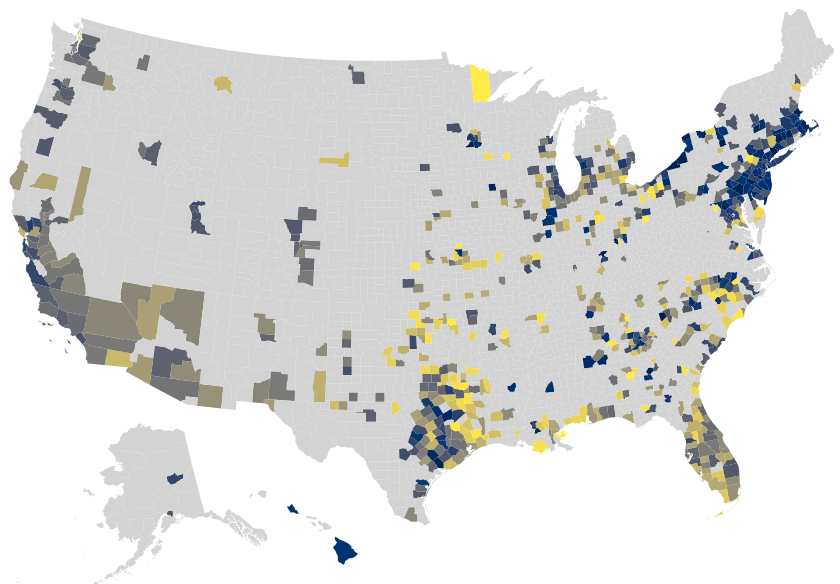

Native American-White Low Birth Weight Rate Ratio $(\mathrm{N}=461)$

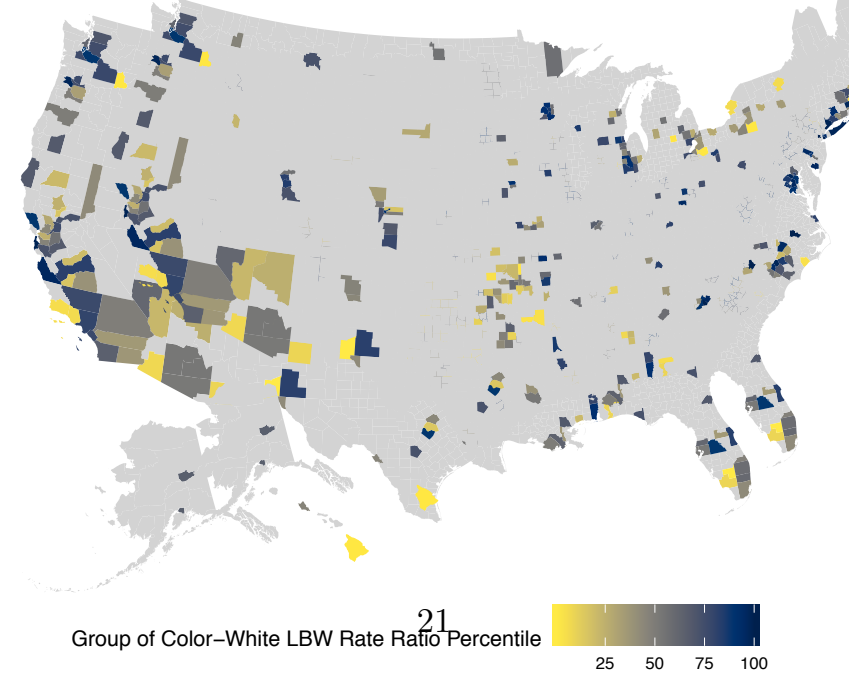

Figure 1: Maps of County-Level Ethnoracial Low Birth Weight Disparities, 2000-2009 

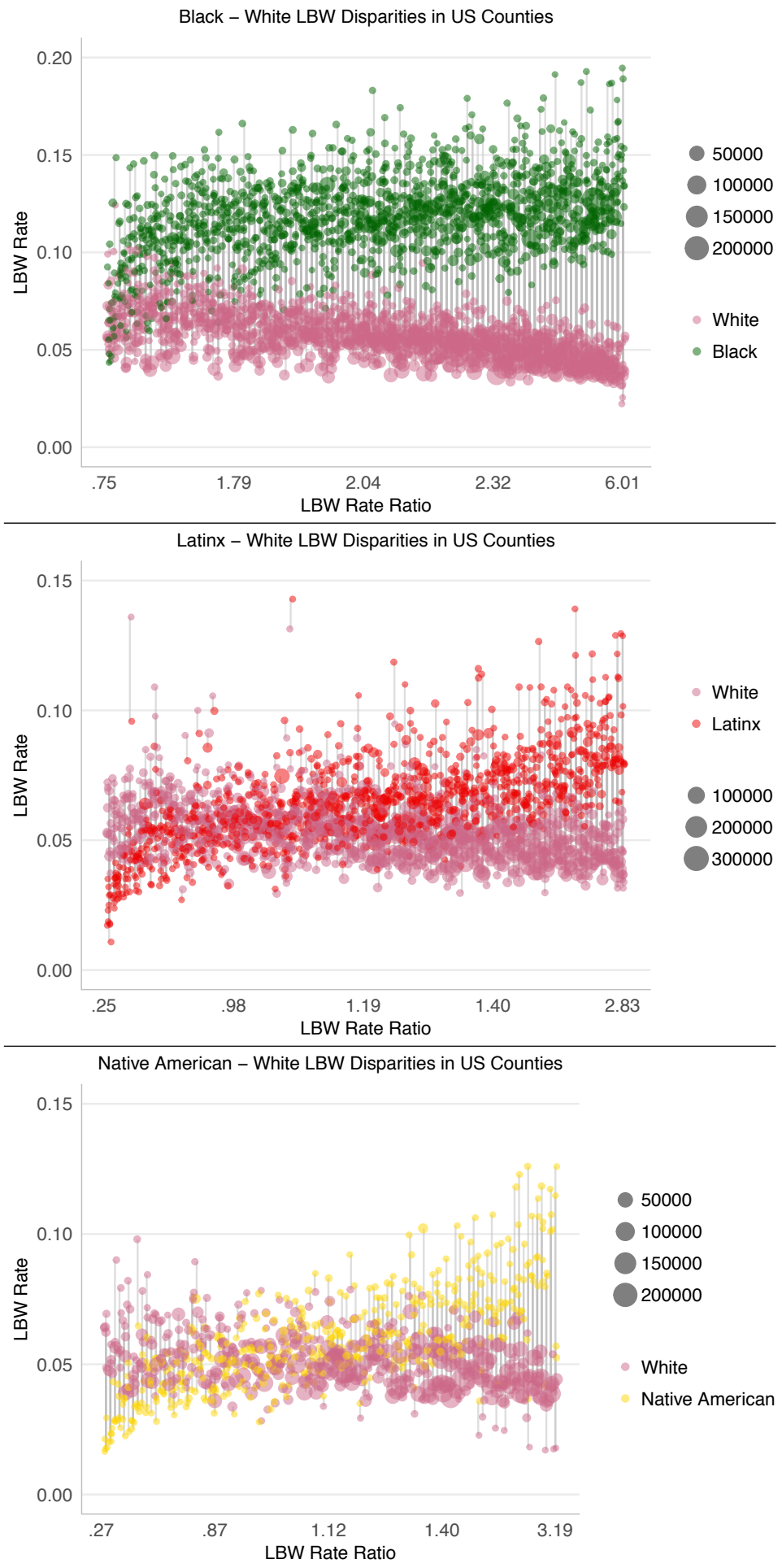

Figure 2: Dot-and-Line Plots of County2Level LBW Disparities in United States Weighted by County Population for Each Ethnoracial Group, 2000-2009.Note: Each dot represents an ethnoracial group's county-level LBW rate (weighted in size by county population). Vertical lines connect each county's White rate and group of color rate, reflecting the rate difference between the two groups. Counties are ordered along the $\mathrm{X}$ axis by the size of the rate ratio disparity (group of color rate/White rate). 


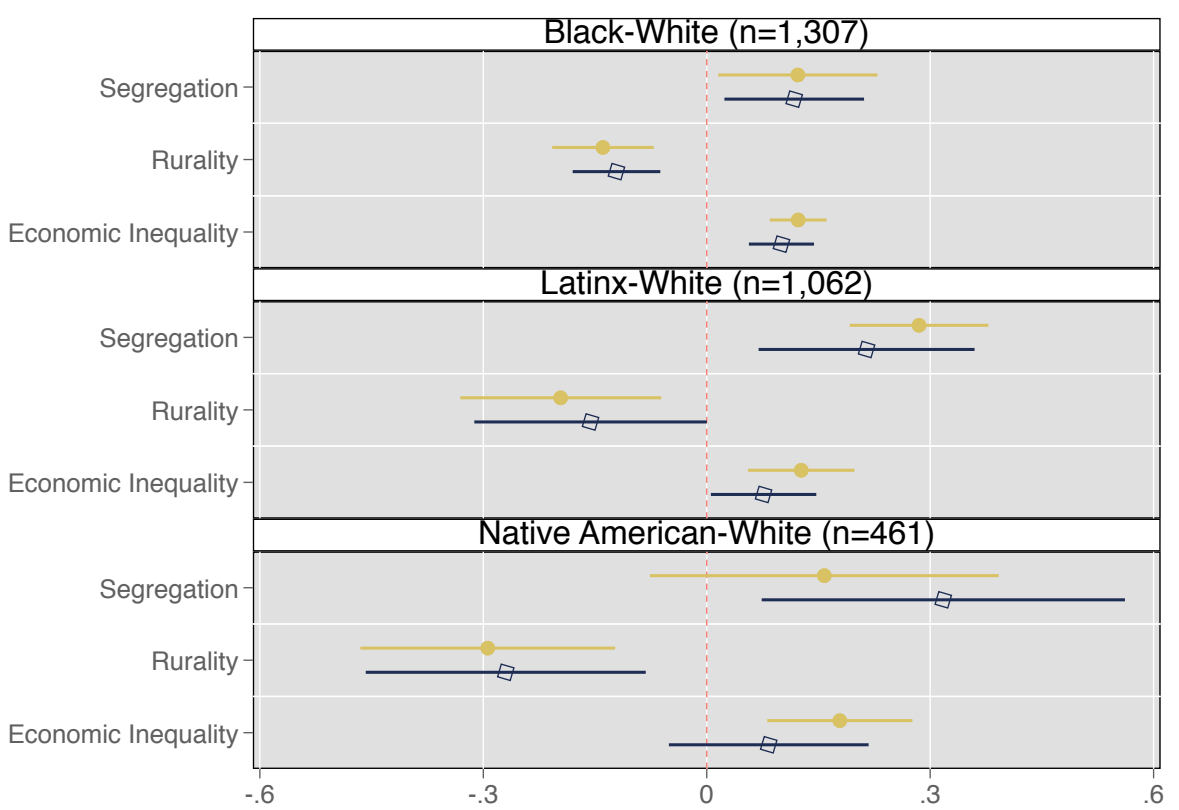

Figure 3: Coefficients and 95\% Confidence Intervals from Adjusted Multivariable Regression Models Predicting County-Level Ethnoracial LBW Disparities. Note: \% rurality scaled (0-1) for ease of visual interpretation. 


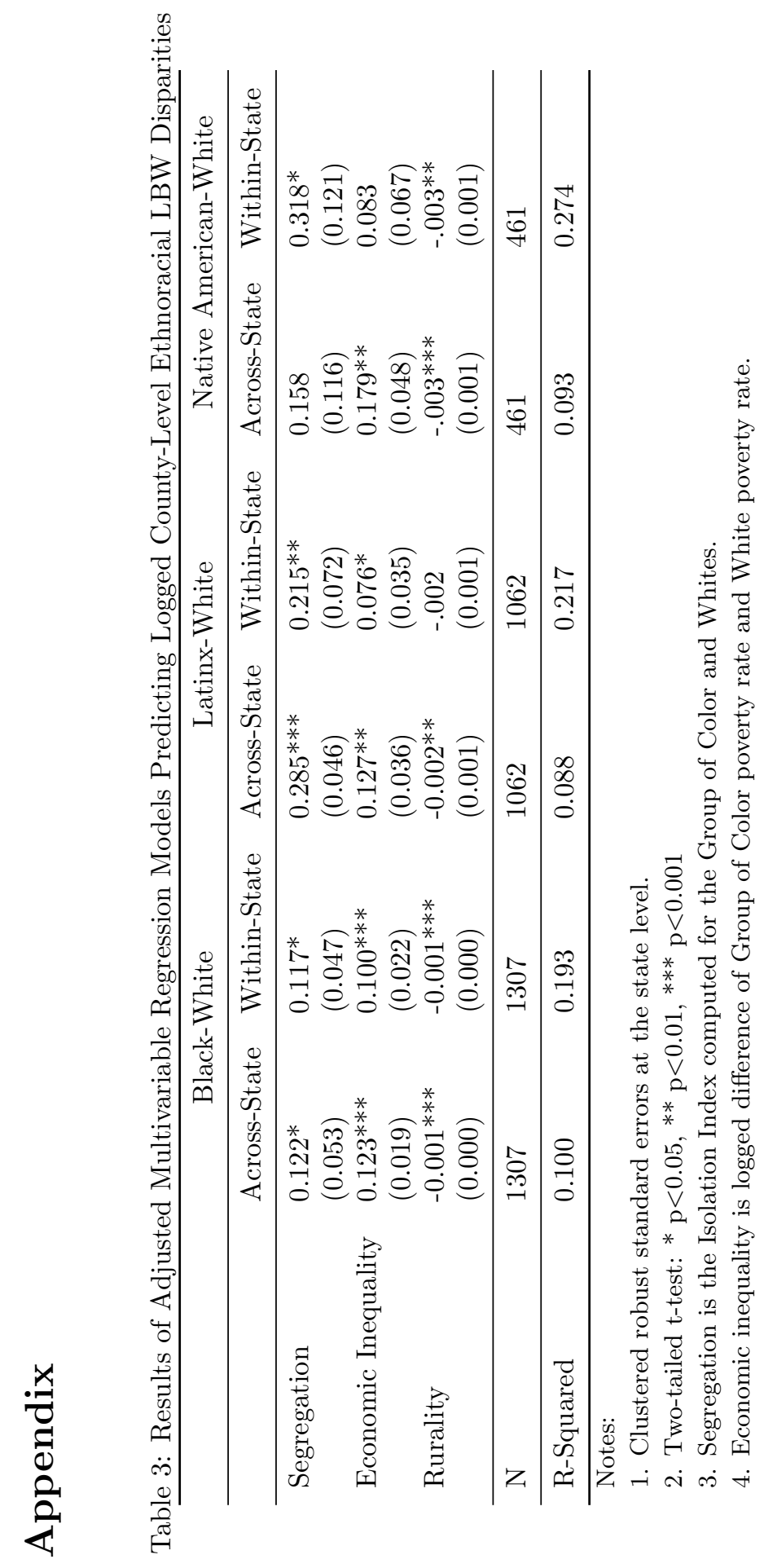




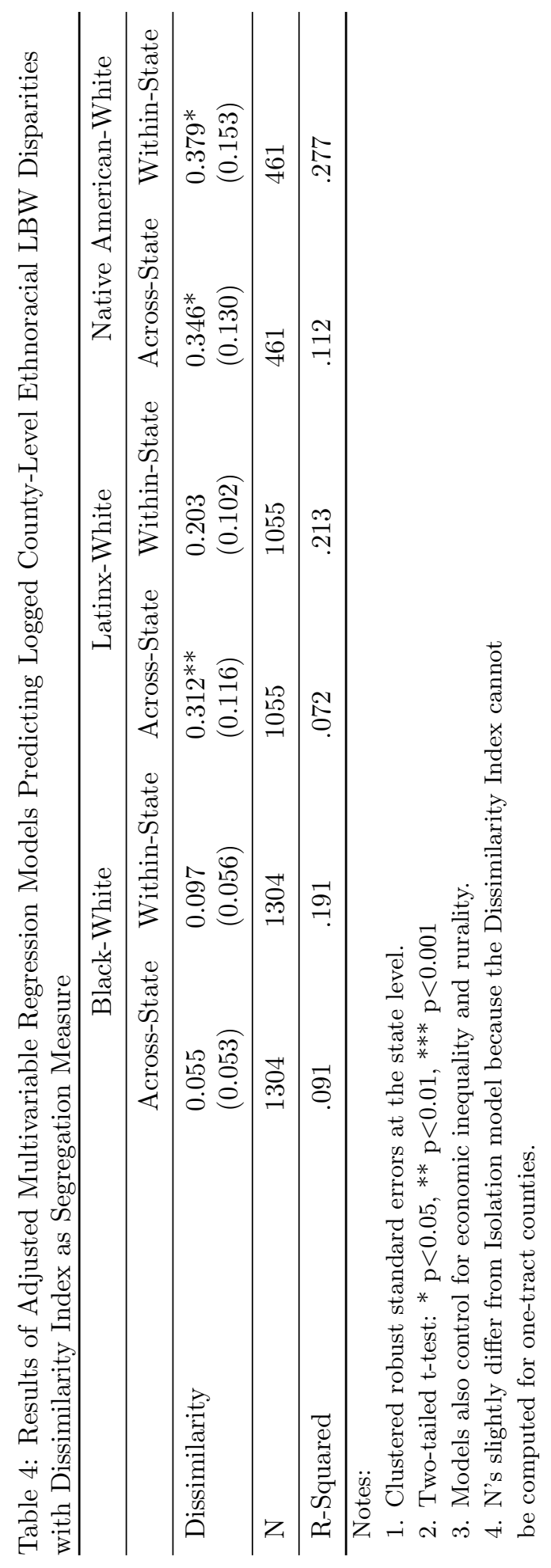




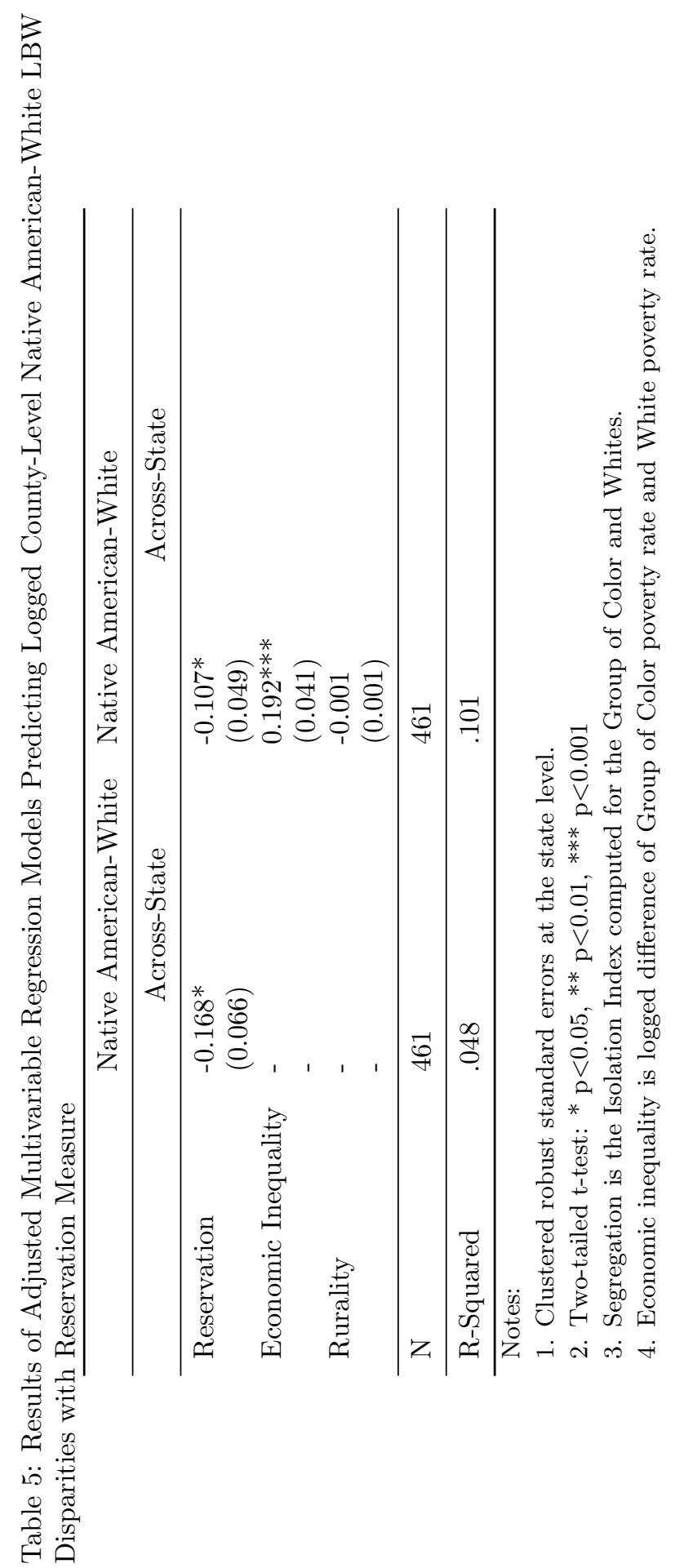




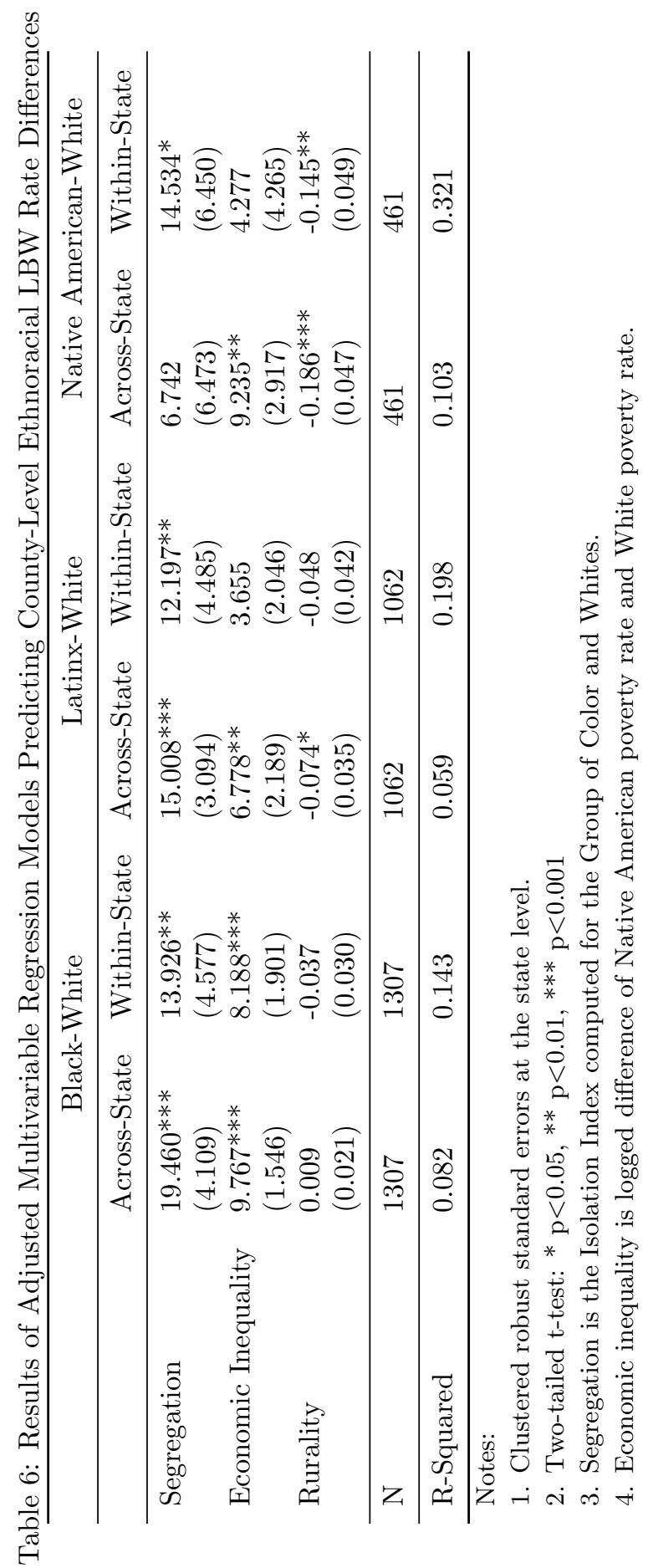

